\title{
Differenzialdiagnose der chronischen Rhinopathie
}

\section{H. RIECHELMANN}

Eine Analyse von 2194 Patienten, die wegen chronischer Rhinopathie an einem rhinologischen Zentrum untersucht wurden, ergab in nur 103 Fällen den sicheren Nachweis einer perennialen allergischen Rhinitis. Die häufigsten Ursachen der geklagten Beschwerden waren anatomische Veränderungen der inneren und äußeren Nase sowie chronische Sinusitiden. Hinzu kamen neurogene Regulationsstörungen, degenerative Veränderungen der Nasenschleimhaut und vergleichsweise selten Neoplasien.

Anatomische Veränderungen der Nase wie Septumdeviationen, Nasenklappenstenosen oder Nasenmuschelhyperplasie wurden im Rahmen der Rhinoskopie festgestellt und mit der Rhinomanometrie funktionell charakterisiert. Chronische Sinusitiden zeigten bei der endoskopischen Untersuchung der Nasenhaupthöhle oft polypös hyperplastische Schleimhautveränderungen oder pathologisches Sekret. Zur Diagnose einer chronischen Sinusitis diente das koronare CT im Knochenfenster.

Die Diagnose der perennialen allergischen Rhinitis erfolgte durch Anamnese, Hauttestungen, den Nachweis von allergenspezifischem IgE im Serum sowie durch eine nasale Allergenprovokation. Die diagnostische Wertigkeit anamnestischer Angaben (s. Tab.), des Hauttests und des RAST war gering. Zum Nachweis einer perennialen Allergie ist demnach ein allergenspezifischer nasaler Provokationstest erforderlich [1, 3, 4]. Nasale Zytologien und die Untersuchung nasaler Zellaktivierungsmarker (ECP, Tryptase, MPO) gaben Aufschluss über die entzündliche Aktivierung der Schleimhaut und dienten der Differenzierung von Rhinitis und Rhinopathie.

Häufigste Ursache neurogener Regulationsstörungen der Nasenschleimhaut war der längerfristige Gebrauch abschwellender Nasentropfen. Die Diagnose der nasalen Hyperreagibilität erfolgte als Ausschlussdiagnose. Eine besondere Form der nasalen Hyperreagibilität äußerte sich als plötzlich auftretende, ca. 10-20 Minuten anhaltende, profuse wässrige Hypersekretion. Hiervon war die Rhinoliquorrhoe abzugrenzen, die meist nach Schädel-Hirn-Trauma oder endonasalen Operationen auftrat. Degenerative Nasenschleimhautveränderungen fielen v.a. durch die Diskrepanz von rhinoskopischem Befund und subjektivem Beschwerdebild auf. Die Nase zeigte sich abnorm weit. Trotzdem wurde über nasales Verstopfungs- gefühl und quälendes zähes Verschleimungsgefühl im Nasenrachen geklagt. Neoplasien machten sich häufig durch einseitge Nasenatmungsbehinderung und blutig tingiertes, fötides Sekret bemerkbar. Berufliche Holzstaubexposition ist ein wesentlicher Risikofaktor. Schon deswegen gehört die Berufsanamnese zur Diagnostik der perennialen Rhinopathie.

Anamnestische Angaben von 168 Patienten mit chronischer Rhinopathie mit und ohne perenniale nasale Allergie.

\begin{tabular}{lll} 
Anamnese & $\begin{array}{l}\text { perenniale } \\
\text { Allergiker }\end{array}$ & nicht-Allergiker \\
\hline pos. Familienanamnese & $29 / 60$ & $56 / 108$ \\
Beschwerden bes. nachts und morgens & $19 / 60$ & $34 / 108$ \\
ganzjährig, Besserung im Urlaub & $32 / 60$ & $52 / 108$ \\
Beschwerden bes. an bestimmten Orten & $20 / 60$ & $28 / 108$ \\
Beschwerden bei Staubexposition & $24 / 60$ & $39 / 108$
\end{tabular}

Im Regelfall traten die genannten pathophysiologischen Veränderungen nicht isoliert, sondern in individuell unterschiedlich gewichteter Kombination auf. Darüber hinaus beeinflussen psychische und emotionale Faktoren die Nasenfunktion. Es lagen demnach aus pathophysiologischer Sicht meist komplexe Mischformen anatomischer, entzündlicher, neurogener und psychischer Kausalfaktoren vor. Aufgabe der Differentialdiagnostik ist es, diese zu identifizieren und deren jeweilige pathophysiologische Bedeutung für das Beschwerdebild des Patienten abzuschätzen.

\section{Literatur}

1. Bachert C, Berdel D, Enzman H et al. Richtlinien für die Durchführung von nasalen Provokationtests mit Allergenen bei Erkrankungen der oberen Luftwege. Allergologie 1990; 2: 53-5.

2. Bascom R. The upper respiratory tract: mucous membrane irritation. Environ Health Perspect 1991; 95: 39-44.

3. Hosen $\mathbf{H}$. Provocative nasal tests for diagnosis of inhalant allergens: correlation with skin tests and clinical symptoms. Ann Allergy 1965; 23: 497-505.

4. Melillo G, Bonini S, Cocco G et al. EAACl provocation tests with allergens. Report prepared by the European Academy of Allergology and Clinical Immunology Subcommittee on provocation tests with allergens. Allergy. 1997; 52 (Suppl): 1-35.

\section{PD Dr. H. Riechelmann}

\section{Clinical evaluation of chronic rhinopathy}

Schlüsselwörter allergische Rhinitis chronische Sinusitis Septumdeviation - nasale Hyperreagibilität

Key words Allergic rhinitis chronic sinusitis nasal septal deviation - nasal hyperreactivity 